\title{
Erratum to: The success of pharmacogenomics in moving genetic association studies from bench to bedside: study design and implementation of precision medicine in the post-GWAS era
}

\author{
Marylyn D. Ritchie
}

Published online: 17 September 2013

(C) Springer-Verlag Berlin Heidelberg 2013

Erratum to: Hum Genet (2012) 131:1615-1626

DOI 10.1007/s00439-012-1221-z

There is an error in the published Table 1 of the original publication. The first column, fourth entry should read as Drug-induced liver injury (amoxicillin-clavulanate) and not Nevirapine-induced rash.

The online version of the original article can be found under doi:10.1007/s00439-012-1221-z.

M. D. Ritchie $(\bowtie)$

Department of Biochemistry and Molecular Biology,

The Huck Institutes of the Life Sciences, Center for Systems Genomics, Eberly College of Science, The Pennsylvania State University, 512 Wartik Laboratory, University Park,

PA 16802, USA

e-mail: marylyn.ritchie@psu.edu

URL: http://ritchielab.com 